\title{
Dynamics of nearly spherical vesicles in an external flow
}

\author{
V. V. Lebedev, K. S. Turitsyn, and S. S. Vergeles \\ Landau Institute for Theoretical Physics, Moscow, Kosygina 2, 119334, Russia
}

(Dated: May 12, 2019)

\begin{abstract}
We analytically derive an equation describing vesicle evolution in a fluid where some stationary flow is excited regarding that the vesicle shape is close to a sphere. A character of the evolution is governed by two dimensionless parameters, $S$ and $\Lambda$, depending on the vesicle excess area, viscosity contrast, membrane viscosity, strength of the flow, bending module, and ratio of the elongation and rotation components of the flow. We establish the "phase diagram" of the system on the $S-\Lambda$ plane: we find curves corresponding to the tanktreading to tumbling transition (described by the saddle-node bifurcation) and to the tank-treading to trembling transition (described by the Hopf bifurcation).
\end{abstract}

PACS numbers: 87.16.Dg, 87.17.Jj, 05.45.a

Vesicles are closed membranes which separate two regions occupied by possibly different fluids. The vesicles are attracting significant attention not only due to their resemblance with biological objects but also because of their importance in different industries such as pharmaceutics where they are used for drug transportation. A natural problem which arises in these applications is understanding of how a single vesicle behaves in an external flow. This non-equilibrium problem has revealed a variety of new physical effects and became a subject of intense experimental and theoretical studies. Laboratory experiments [1, 2, 3, 4, 5] have shown that the vesicles immersed in a shear flow exhibit at least two qualitatively different types of behavior, either tank-treading or tumbling motion. In the tank-treading regime a vesicle shape is stationary, it is ellipsoid oriented at an angle with respect to the shear flow. In the tumbling regime the vesicle experiences periodic flipping in the shear plane. A novel type of behavior: trembling, discovered in the work [6] is an intermediate regime between tank-treading and tumbling in which a vesicle trembles around the flow direction.

Constructing a phase diagram for all these regimes depending on the external parameters is a challenging and an extremely difficult task because the problem in consideration is both strongly non-linear and non-equilibrium. As long as no analytic solution of this problem exists theoretical studies were based either on numerical simulations or on some approximations allowing analytical treatment. Numerical investigations of this problem involved several different computational schemes, including boundary element method [7], mesoscopic particle-based approximation [8, 9, 10, 11, 12], and an advected field approach [13]. These approaches have shown qualitative agreement with experiments however did not solve the problem of constructing the vesicle dynamics phase diagram completely. Analytical studies of the problem can be divided in two major classes. In the first one [9, 14, 15], phenomenological models of a vesicle dynamics based on the classical work of Keller and Skallak [16] were proposed and proved themselves to be rather efficient in explaining the experiments. In the second series of works [17, 18, 19, 20] the studies focused on quasi-spherical vesicles whose shape can be parameterized by spherical harmonics expansion. A pertur- bation scheme around the Lamb solution for spherical particle in external flow allows one to derive the dynamic equations for the shape and the orientation of a vesicle and to investigate them analytically. In this letter we propose a natural extension of the theory developed for quasi-spherical vesicles by accounting higher-order expansion terms. We show that these additions produce a qualitative change in the phase diagram and make it significantly more complicated. The resulting diagram which is the main result of this paper contains all three types of vesicle behavior which were observed experimentally. In our work we analyze how the vesicle dynamics depends on different control parameters, such as viscosity contrast, vesicle excess area, internal membrane viscosity, strength of the flow, and ratio of the elongation and rotation components of the flow. We analyze also the vesicle orientation in the tank treading regime.

Speaking about membranes we have in mind lipid bilayers. Physical properties of such objects have been extensively studied both experimentally and theoretically (see e.g. the books [21, 22, 23] and the reviews [24]). There are several features of the membranes which are important for our analysis. First, we assume that the membrane is in a fluid state (is a $2 d$ liquid), which is typical of the lipid bilayers under normal conditions. Second, we assume that the vesicle has an excess area, that enables one to treat the vesicle membrane as incompressible. Third, we assume that the membrane is impermeable to the surrounding liquids, the condition is usually well satisfied in experiment. Finally, we take into account the membrane internal viscosity, which could play an essential role, say, in a vicinity of the lipid-bilayer melting point [25] (see also Refs. [9, 12]).

We assume that the vesicle size is much larger than the membrane thickness. Then, in the main approximation, the vesicle membrane can be treated as infinitesimally thin, that is as a $2 d$ object immersed into a $3 d$ fluid. By other words, in the limit the vesicle can be considered as an interface separating two generally different fluids. The two properties, the membrane incompressibility and impermeability, imply that both the vesicle volume $\mathcal{V}$ and the membrane area $\mathcal{A}$ are conserved. The excess area can be characterized by a dimensionless factor $\Delta$, which is defined as $\mathcal{A}=(4 \pi+\Delta) r_{0}^{2}$, where $r_{0}$ is a 
vesicle "radius" determined by its volume: $\mathcal{V}=4 \pi r_{0}^{3} / 3$. The excess area is non-negative, $\Delta \geq 0$, and the minimal value $\Delta=0$ corresponds to ideal spherical geometry.

The membrane free energy can be written as the following integral over the membrane position [26, 27, 28, 29]

$$
\mathcal{F}=\int d A\left(\sigma+\frac{\kappa}{2} H^{2}\right),
$$

where $\sigma$ is the membrane surface tension, $H$ is its mean curvature, and $\kappa$ is Helfrich module. The last term in Eq. (1) describes energy related to membrane bending distortions. Note that the surface tension $\sigma$ is a quantity adjusting to other membrane parameters (similarly to pressure in incompressible fluid) to ensure a given value of the membrane area $A$.

The membrane moves together with surrounding fluid that is the velocity field $v$ is continuous on the membrane and $v$ determines the membrane velocity as well as the fluid velocity. We divide the flow near the vesicle into two parts: an external flow which would occur in the fluid in the absence of the vesicle, and an induced flow which is excited as a result of the vesicle reaction to the external flow. We assume that a characteristic scale of the external flow is much larger than the vesicle size. Then the external flow velocity $\boldsymbol{V}$ near the vesicle can be approximated by a linear profile. Generally, the external flow has both the strain (elongation) and the rotational contributions: $\partial_{i} V_{k}=s_{i k}+\epsilon_{i k j} \omega_{j}$, where $\hat{s}$ is the (symmetric) strain matrix and $\boldsymbol{\omega}$ is the angular velocity vector. The strain can be characterized by its strength $s$, defined as $s^{2}=\operatorname{tr} \hat{s}^{2} / 2$. Note that for a shear flow $s=\omega=\dot{\gamma} / 2$, where $\dot{\gamma}$ is the shear rate.

We examine nearly spherical vesicles, that is the excess area parameter $\Delta$ is regarded to be small. Then it is natural to describe the vesicle shape (membrane position) as $r=r_{0}[1+u(\theta, \varphi)]$ where $r, \theta, \varphi$ are spherical coordinates in the reference system with origin chosen at the vesicle center. The quantity $u$ is a dimensionless displacement characterizing deviations of the membrane shape from a spherical one. For small $\Delta$ the value of $u$ can be estimated as $u \sim \sqrt{\Delta}$. Therefore in our scheme $u \ll 1$ and one can formulate a perturbation theory in the parameter.

We assume that both interior fluid and exterior one are Newtonian and that Reynolds number is vanishingly small, so the fluids can be described in terms of Stokes equation. We assume also that an adiabaticity condition $\max \left\{s, \kappa /\left(\eta r_{0}^{3}\right)\right\} \ll$ $\eta /\left(\varrho r_{0}^{2}\right)$ is satisfied where $\eta$ is viscosity and $\varrho$ is mass density of the exterior fluid. The same condition is assumed for the interior fluid. Then the flow inside and outside the vesicle can be treated as instantaneously adjusted to the vesicle motion. In this case it is possible to establish a closed dynamic equation for the membrane displacement $u$. For the purpose one should find the velocity field $v$ inside and outside the vesicle at a given displacement $u(\theta, \varphi)$ and then equate $\partial_{t} u$ to the membrane normal velocity. To find the velocity field one should solve the Stokes equation with boundary conditions dictated by the membrane incompressibility and by the momentum balance that includes membrane forces determined by the energy [1] [17, 30, 31] and membrane viscosity. To realize the program for a nearly spherical vesicle one can use a generalization of the Lamb scheme applicable to a spherical body, see Ref. [32]. As a result, one finds the dynamic equation for $u$ as a series in $u$.

In the main approximation in $u$ one obtains

$$
\hat{a}\left(\partial_{t}-\omega \partial_{\varphi}\right) u=10 s_{i j} \frac{r_{i} r_{j}}{r^{2}}-\frac{1}{\eta r_{0}^{3}} \frac{\delta \mathcal{F}^{(3)}}{\delta u},
$$

where $\hat{a}$ is some dimensionless operator, reflecting all viscous mechanisms, the $Z$-axis of our reference frame is chosen to be directed opposite to the angular velocity $\boldsymbol{\omega}$, and $\mathcal{F}^{(3)}$ is an expansion up to third order in $u$ of the free energy (1). Note that the elongation and the rotation parts of the external flow are separated in Eq. (2): the angular velocity $\boldsymbol{\omega}$ extends the time derivative whereas the strain determines the non-uniform term playing a role parallel to the free energy derivative. For an external shear flow $s_{i j} r_{i} r_{j} / r^{2}=(\dot{\gamma} / 2) \sin ^{2} \theta \sin (2 \varphi)$, where $X$-axis is directed along the velocity.

The two differences between the equation (2) and the analogous equations obtained by Misbah [19] and Vlahovska et. al. [20] are the inclusion of membrane viscosity and third order expansion term of Helfrich energy. An account of the third order term changes the phase diagram completely since the second order term becomes vanishingly small (due to the surface tension adjustment) in the vicinity of the tumbling to tanktreading transition region predicted in Refs. [19, 20]. An importance of the third-order term is discussed also by Noguchi and Gompper [15].

We take into account only the main contribution to $u$ determined by second order angular harmonics. It is justified by the smallness of $\Delta$ and by the fact that the non-uniform term in the right-hand side of Eq. (2) is a linear combination of second order angular harmonics. The operator $\hat{a}$ in the case is reduced to a constant

$$
a=\frac{16}{3}\left(1+\frac{23}{32} \frac{\tilde{\eta}}{\eta}+\frac{\zeta}{\eta r_{0}}\right)
$$

where $\tilde{\eta}$ is viscosity of the interior fluid and $\zeta$ is membrane viscosity. Note that the expression (3) includes the viscosity contrast $\tilde{\eta} / \eta$. After passing to the variable $u / \sqrt{\Delta}$ the equation (2) acquires a self-similar form containing the parameters $\sqrt{\Delta} a \omega / s, s \eta r_{0}^{3} /(\kappa \Delta)$ and some dimensionless parameters characterizing ratios of the eigenvalues of the matrix $\hat{s}$ and mutual orientation of the vorticity vector $\boldsymbol{\omega}$ and the main axes of the matrix $\hat{s}$.

Below, we consider a plain flow where the external velocity $V$ lies in $X-Y$ plane. In the case the equation for $u / \sqrt{\Delta}$ depends on two dimensionless parameters which can be chosen as

$$
\Lambda=\frac{\sqrt{3}}{4 \sqrt{10 \pi}} \frac{\sqrt{\Delta} a \omega}{s}, \quad S=\frac{14 \pi}{3 \sqrt{3}} \frac{s \eta r_{0}^{3}}{\kappa \Delta} .
$$

In the case of weak strains, $S \ll 1$, the vesicle conserves its equilibrium shape, and in the case of strong strains, $S \gg 1$, 


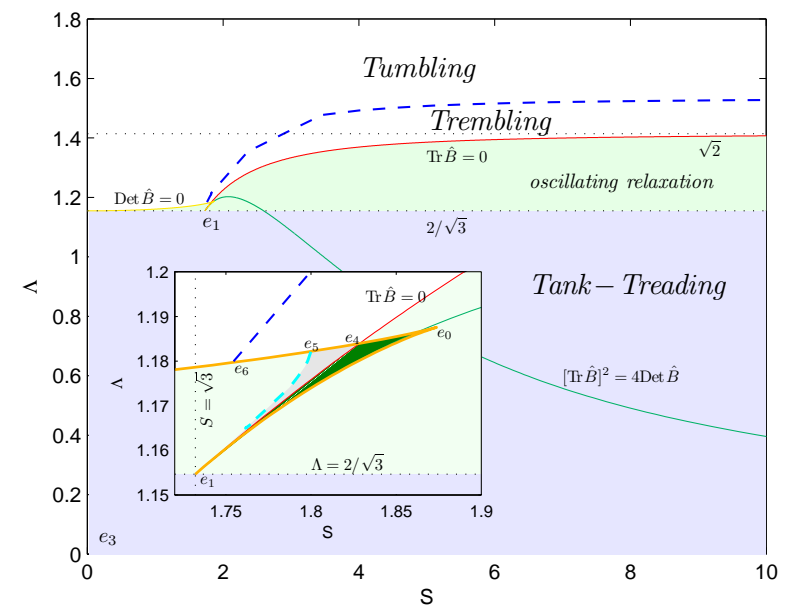

FIG. 1: Phase diagram on the $S-\Lambda$ plane.

the vesicle shape is determined by the external flow. The parameter $\Lambda$ determines an effectiveness of the rotational part of the external flow. Note that our theory is applicable, particularly, to purely elongation flow, where $\omega=0$ and, consequently, $\Lambda=0$.

For the plain flow, one can further reduce description of the vesicle shape which is dependent on two parameters, $\Theta$ and $\Phi:$

$$
u=\frac{\sqrt{5 \Delta}}{4 \sqrt{2 \pi}}\left[\frac{\sin \Theta}{\sqrt{3}}\left(1-3 \cos ^{2} \theta\right)+\cos \Theta \sin ^{2} \theta \cos 2(\varphi-\Phi)\right] .
$$

Here, the axes $X$ and $Y$ are chosen to be directed along the principal axes of the strain matrix $\hat{s}$. Note that the "angles" $\Phi, \Theta$ determine the main axis direction of the vesicle projection to the $X-Y$ axis. In the limit $\Delta \ll 1$ the inclination angle of the vesicle projection main axis is $\varphi^{*}=\Phi$ for $\cos \Theta>0$ and $\varphi^{*}=\Phi+\pi / 2$ otherwise. The parameter $\Theta$ determines also the vesicle shape and can be directly related to the parameter $R=\cos \Theta$ introduced in Ref. [19]. In terms of the variables $\Phi$ and $\Theta$, Eq. (2) is reduced to a couple of equations

$$
\begin{array}{r}
\tau \partial_{t} \Phi=\frac{S}{2}\left[\frac{\cos (2 \Phi)}{\cos \Theta}-\Lambda\right], \\
\tau \partial_{t} \Theta=-S \sin \Theta \sin (2 \Phi)+\cos (3 \Theta), \\
\text { where } \quad \tau=\frac{7 \sqrt{\pi}}{12 \sqrt{10}} \frac{a \eta r_{0}^{3}}{\kappa \sqrt{\Delta}} .
\end{array}
$$

The tank treading vesicle motion corresponds to stable stationary points of the equations (5)6. Equating to zero the right hand sides of the equations one finds relations determining a stationary point at given parameters $S$ and $\Lambda$. To investigate stability of the point one should linearize the equations 5 , (6) near the point to obtain $\tau \partial_{t}(\delta \Theta, \delta \Phi)=\hat{B}(\delta \Theta, \delta \Phi)$. The point is stable, if both eigenvalues of the matrix $\hat{B}$ have negative real parts. Thus the stability conditions are $\operatorname{tr} B<0$ and $\operatorname{det} B>0$. Regions in the $S-\Lambda$ plain where stable points exist are indicated in Fig. 1 by blue color. Note that there exists an additional region of stationary points which are stable in terms of $\Theta$ and $\Phi$. However, a stability investigation in the framework of Eq. (2) shows that the points are unstable in the extended space where the spherical harmonics $Y_{2, \pm 1}(\theta, \phi)$ are involved into play. Therefore we do not analyze the points.

If $\Lambda$ is increased at a given value of $S$ then first the stable point is in the domain where $\Phi>0$. At the boundary of the domain, at $\Lambda=2 / \sqrt{3}$, the parameters $\Phi$ and $\Theta$ come to the values $\Phi=0, \Theta=\pi / 6$. Then the stable point moves into the domain, corresponding to $\Lambda>2 / \sqrt{3}$ and $\Phi<0$. The green line in Fig. 1 is determined by the condition $(\operatorname{tr} B)^{2}=4 \operatorname{det} B$, it separates regions where oscillatory and damping regimes of approaching the stable point occur. The boundary of the stability domain can be determined either by the condition $\operatorname{det} B=0$ or $\operatorname{tr} B=0$. The corresponding lines are depicted in Fig. 1 by yellow and red lines, respectively. The first case is realized at small $S$ whereas the second case is realized at large $S$. At increasing $\Lambda$ a Hopf bifurcation occurs at the red line whereas a saddle-node bifurcation occurs at the yellow line. A limit cycle is realized in the system for points above the red and the yellow lines (that is at large $\Lambda$ ). Following the work [6] we distinguish tumbling and trembling regimes. In our terms, trembling corresponds to a limit cycle where $\varphi$ varies in a restricted interval whereas tumbling means an increase of $\varphi$ by $2 \pi$ during the cycle. The dashed blue line in Fig. 1 separates the two regimes, trembling is realized below the line. A possibility of such regime is discussed also by Misbah [19]. The sequence of the vesicle states predicted by our theory corresponds to one observed in experiment [6]. Qualitative the picture is also similar to the one observed in recent numerical simulations by Noguchi and Gompper [15]. Note that at infinitely large $\tilde{\eta}$ or $\zeta$ a solid ball behavior of the vesicle should be observed which is tumbling as Jeffery established [33]). In our scheme large $\tilde{\eta}$ or $\zeta$ implies large $\Lambda$, corresponding to tumbling region, indeed.

A special point $S=\sqrt{3}, \Lambda=2 / \sqrt{3}$, where two transition lines terminate, is analogous to some extend to fluid critical point. Say, frequency of the Hopf bifurcation behaves $\propto \sqrt{S^{2}-3}$. Similar "smoothing" of the saddle-node bifurcation near the point occurs. A complicated phase structure is observed near the special point, its narrow vicinity is plotted in the insertion to Fig. 1] The green region corresponds to coexistence of two stable points. There exists also a region where the stable point and the limit cycle coexist. The limit cycle becomes unstable at the dashed green line.

Thermal fluctuations of the vesicle shape can be investigated in spirit of Refs. [17, 34, 35] (see also the book [36]). The fluctuations produce isotropic Brownian motion on the sphere, defined by the "angles" $\Theta$ and $\Phi$ with the characteristic angular diffusion coefficient $D \sim k T /\left(\eta r_{0}^{3} \Delta\right)$, where $T$ is temperature. In the tank-treading regime and strong external flow, $S \gg 1$, fluctuations induced by this diffusion can be estimated as $(\delta \Theta)^{2} \sim(\delta \Phi)^{2} \sim D \tau / S$ if $\Lambda<2 / \sqrt{3}$ and as $(\delta \Theta)^{2} \sim(\delta \Phi)^{2} \sim D \tau$ if $\Lambda>2 / \sqrt{3}$, where $\tau$ is defined by Eq. (7). For weak external flow, $S \ll 1$, fluctu- 
ations of vesicle shape are given by estimate $(\delta \Theta)^{2} \sim D \tau$, whereas vesicle orientation experiences more stronger fluctuations, $(\delta \Phi)^{2} \sim D \tau / S$. The thermal fluctuations play an essential role near the tank-treading to tumbling (or tanktreading to trembling) transition "smearing" it. Results of detailed investigation of this phenomenon will be published elsewhere.

To conclude, we have theoretically investigated the vesicle dynamics in an external stationary flow. The general scheme based on solving the $3 d$ hydrodynamic (Stokes) equations with boundary conditions posed on the membrane enabled us to analyze in detail dynamical properties of nearly spherical vesicles. We constructed the phase diagram of the system in terms of two dimensionless parameters (4) which are combinations of physically observed quantities. We demonstrated that there are two different regimes realized in weak and strong external flows. In weak flows the vesicle shape is close to an equilibrium one which is a prolate ellipsoid and a role of the external flow is reduced mainly to an orientation of the ellipsoid. Then the tank-treading to tumbling transition occurs which can be described by a saddle-node bifurcation. In strong flows the vesicle shape and orientation are determined by the flow. Then the tank-treading to trembling transition occurs which can be described by a Hopf bifurcation. The two transition curves are separated by a point vicinity of which needs a special consideration.

The authors acknowledge numerous discussions of the experiments with V. Steinberg and V. Kantsler. This work has been partially supported by RFBR. KT and SV also acknowledge the financial support from "Dynasty" and RSSF foundation.

[1] K. H. De Haas, C. Blom, D. E. Van, D., M. H. G. Duits, J. Mellema, Phys. Rev. E 56, 7132 (1997).

[2] N. Shahidzadeh, D. Bonn, O. Aguerre-Chariol, J. Meunier, Phys. Rev. Lett. 81, 4268 (1998).

[3] M. Abkarian, C. Lartigue, and A. Viallat, Phys. Rev. Lett. 88, (2002).

[4] V. Kantsler and V. Steinberg, Phys. Rev. Lett., 95, 258101 (2005).

[5] M. A. Mader, V. Vitkova, M. Abkarian, A. Viallat, and T. Podgorski, Eur. Phys. J. E 19, 389 (2006).

[6] V. Kantsler and V. Steinberg, Phys. Rev. Lett., 96, 036001 (2006).

[7] M. Kraus, W. Wintz, U. Seifert, and R. Lipowsky, Phys. Rev. Lett. 77, 3685 (1996).S. Sukumaran and U. Seifert, Phys. Rev.
E, 64, 011916 (2001).

[8] H. Noguchi and M. Takasu, Phys. Rev. E 65, (2002).

[9] H. Noguchi and G. Gompper, Phys. Rev. Lett., 93, 258102 (2004)

[10] H. Noguchi and G. Gompper, Journal of Physics Condensed Matter, 17, S3439 (2005)

[11] H. Noguchi and G. Gompper, Proc. Nat. Ac. Sci., 102, 1415914164 (2005)

[12] H. Noguchi and G. Gompper, Phys. Rev. E ., 72, 011901 (2005)

[13] T. Biben, C. Misbah, Eur. Phys. J B 29, 311 (2002). T. Biben, C. Misbah, Phys. Rev. E, 67, 031908 (2003). J. Beaucourt, F. Rioual, T. Seon, T. Biben, and C. Misbah, Phys. Rev. E 69, 011906 (2004). T. Biben, K. Kassner, and C. Misbah, Phys. Rev. E 72, (2005).

[14] F. Rioual, T. Biben, and C. Misbah, Phys. Rev. E 69, (2004).

[15] H. Noguchi and G. Gompper, Swinging and Tumbling of Fluid Vesicles in Shear Flow, arXiv:cond-mat/0611382

[16] S. R. Keller and R. Skalak, J. Fluid Mech. 120, 27 (1982).

[17] U. Seifert, Eur. Phys. J. B, 8, 405 (1999).

[18] P. Olla, Physica A, 278, 87-106 (2000).

[19] C. Misbah, Phys. Rev. Lett., 96, 028104 (2006).

[20] P. M. Vlahovska and R. S. Gracia, Phys. Rev. E 75, (2007).

[21] Physics of Amphiphilic Layers, J. Meuner, D. Langevin, and N. Boccara, Springer Proceedings in Physics, 21, Springer-Verlag, Berlin, 1987.

[22] S. A. Safran and N. A. Clark, Physics of Complex and Supermolecular Fluids, Wiley, NY, 1987.

[23] D. Nelson, T. Pvian, and S. Weinberg, Statistical Mechanics of Membranes and Surfaces, World Scientific, NY, 1989.

[24] A. M. Bellocq et. al., Adv. Colloid Interface Sci. 20, 167 (1984). G. Porte, et. al., Physica A176, 168 (1991).G. Porte, et. al. J. Phys. II 4, 8649 (1992).

[25] R. Dimova, B. Pouligny, and C. Dietrich, Biophys. J. 79, 340 (2000).

[26] P. B. Canham, J. Theor. Biol. 26, 61 (1970).

[27] W. Helfrich, Z. Naturforsch. A 28c, 693 (1973).

[28] E. Evans, Biophys. J. 14, 923 (1974).

[29] W. Helfrich, Z. Naturforsch B103, 67 (1975).

[30] Ou-Yang Zong-Can and W. Helfrich, Phys. Rev. A39, 5280 (1989).

[31] V. V. Lebedev and A. R. Muratov, ZhETF 95, 1751 (1989) [Sov. Phys. JETP 681011 (1989)].

[32] H. Lamb, Hydrodynamics (Cambridge Uiversity Press, Cambridge, England, 1932), 6th ed.

[33] G. B. Jeffery, Proceedings of the Royal Society of London. Series A, 102, No. 715, 161-179 (1922).

[34] E. I. Kats, V. V. Lebedev, and A. R. Muratov, Nearly spherical vesicles: Shape fluctuations, Pis'ma v ZhETF, 63, 203 (1996) [JETP Lett. 63, 216-221 (1996)].

[35] K. S. Turitsyn, submitted to ZhETF, arXiv:nlin.CD/0501025

[36] E. I. Kats and V. V. Lebedev, Fluctuational Effects in the Dynamics of Liquid Crystals Springer-Verlag, NY, 1993. 